\title{
Core-Shell Magnetic Polymer Colloids for Self assembled Multilayers Thin Films and Ultrasensitive Molecular Imaging
}

\author{
HIRA FATIMA ${ }^{1}$, Naveed Ahmed ${ }^{2}$, Nasir Ahmad $^{2}$, and Abdelhamid Elaissari ${ }^{2}$ \\ ${ }^{1}$ Kangwon National University \\ ${ }^{2}$ Affiliation not available
}

June 2, 2020

\begin{abstract}
In the present work, novel core-shell polymer magnetic colloids are prepared and employed as a potential candidate in ultrasensitive molecular imaging. The synthesized colloids possess high colloidal stability, magnetization (43.85 emu g-1) and uniformity of particle size with an average diameter of $527 \mathrm{~nm}$. FTIR spectrum of magnetic emulsion shows two characteristic peaks at 570 and $630 \mathrm{~cm}-1$ which related to Fe-O vibration bands. Both T1 and T2 relaxation times were successfully measured. Based on signal intensities, the prepared colloids observed to perform as better T2 weighted contrast agents. The T2-weighted MR images showed significant signal intensity reduction and contrast darkening. Moreover, the prepared colloids were also employed to fabricate thin films via a facile method of layer by layer self-assembled multilayers (LBL-SAMU) to explore their potential application in imaging. Uniform particle size distribution with spherical morphologies was obtained for the fabricated bilayers. In addition, a $20 \%$ increment in iron contents was observed for 5-15 bilayer thin films decorated with film colloidal particles. The reported work opens new avenues for designing powerful T2 contrast agents in various biomedical applications such as analysis of biomacromolecules, diagnostics, and therapy.
\end{abstract}

\section{Introduction}

Magnetic resonance imaging (MRI) is one of the most potent techniques for diagnosis and detection in biological molecular imaging. MRI provides the visibility to demarcate most of the tissues and acquire threedimensional images of whole tissue along with high spatial resolution without using radiotracers. The image contrast can be enhanced by a negative-positive contrast agent that sharpens the MRI image by affecting the spin moment of the protons. The introduction of these agents advances the characterization possibilities by giving heightened signals of specified cells/tissue when compared with the other cells or diseased ones [1-3]. MRI uses the property of protons of hydrogen nuclei in water molecules to align with and precess about an applied static magnetic field. The precessing protons can be disturbed by radiofrequency pulses "RF-pulses", and the mechanism through which they realigned to their original state can be recorded to get an image. The contrast originates from the differences in spin relaxation kinetics of hydrogen protons along with the transverse (spin-spin relaxation time $\mathrm{T}_{2}$ ) and longitudinal (spin-lattice relaxation time $\mathrm{T}_{1}$ ) planes of the externally applied magnetic field to the sample. Negative contrast agents may shorten the transverse relaxation giving darkening of contrast images whereas positive contrast agents interacting with water protons may give a brighter image with shortening of longitudinal relaxation time. Since, contrast generated under MRI is dependent on the physicochemical nature of the targeting tissues, relaxation times of protons, and proton density of water molecules [3-8]. Contrast agents employed for MRI analysis are classified according to the relaxation mechanism they followed when the $\mathrm{RF}$-pulse is removed. To date, $\mathrm{T}_{1}$ weighted contrast agents (gadolinium), and $\mathrm{T}_{2}$ weighted contrast agents (superparamagnetic iron oxide-based materials) are commonly synthesized these days to be used in testing. Superparamagnetic materials can produce promptly enhanced proton nuclei relaxation than paramagnetic materials. Consequently, even at a lower dosage, these 
can target large surface areas. $\mathrm{T}_{2}$ contrast agents based on magnetic colloidal suspensions are frequently termed as USPIO (ultrasmall superparamagnetic iron oxide) [9-11]. The magnetism, magnetic behavior, and magnetic properties of fine colloidal suspensions have been a dynamic field of study for over fifty years. Generally, magnetic colloids or ferrofluids are synthesized by a one-step process involving co-precipitation of $\mathrm{Fe}^{+2}$ and $\mathrm{Fe}^{+3}$ precursors in aqueous solutions [12]. Key parameters for the communications with biological tissues are their magnetic properties, surface functionality, and solubility. Depending on the applications, the efficacy of magnetic colloids can thus be altered to vary their contrast capability [13-16].

In the present study, core-shell magnetic polymer colloids (CSMPC) with average particle size $527 \mathrm{~nm}$ have been prepared using a simple seeded emulsion polymerization technique, followed by decoration of thin films via LBL self-assembly. The final prepared colloidal particles were then incorporated and tested as an MR contrast agent by measuring the transverse and longitudinal relaxation times $T_{2}$ and $T_{1}$ of protons present in the colloidal suspension using a $1.5 \mathrm{~T}$ clinical scanner. Furthermore, in this work, we present a novel technique for the fabrication of contrast agents based thin films of colloids to obtain in vitro MRI images to open the ways for lab-on-chip technology for diagnostics. Fabrication of films by electrostatic attraction of oppositely charged ions via layer by layer self-assembly (LBL-SAMU) using eco-friendly colloids suspensions has been broadly explored numerous biomedical applications. LBL-SAMU technique is a highly efficient, versatile, cost-effective, and facile technique to fabricate robust thin films of colloids by immersing the substrate in colloidal suspensions [17-19]. Each immersion cycle in oppositely charged species is capable of uniform particle distribution and can induce reproducible growth of thin films [20]. The salient characteristics of such thin-film development include their future potential in various applications, no pre-injection requirement, efficient contrast, biomolecule capturing, proficient cell labeling, and assistance to study dynamics of drug delivery and distribution within the body.

\section{Materials and methods}

\subsection{Materials}

1,4-Divinylbenzen (DVB, 80\%) and glycidyl methacrylate (GMA, 97\%) monomers, potassium persulfate (KPS) water-soluble initiator, and sodium dodecyl sulfate (SDS) stabilizing agent were purchased from Aldrich Company. The monomers were purified from the inhibitors by washing with a $5 \% \mathrm{NaOH}$ solution before use. Magnetic emulsion (Emul-500, ADEM Tech, France) with solid content $8 \%$ and an average hydrodynamic size of $450 \mathrm{~nm}$ (as measured by Zetasizer) was used [57]. Milli-Q water was used in all experiments as the dispersion medium.

\subsection{Methods}

\subsubsection{Synthesis of epoxy-functionalized magnetic colloidal particles}

Magnetic latex particles with a magnetic core and epoxy-functionalized polymer shell were prepared by the co-polymerization of DVB and GMA monomers at DVB: GMA volume ratio (2:1). The reaction was conducted by batch emulsion polymerization in the presence of o/w magnetic emulsion droplets as the seed. Briefly, DVB and GMA monomers were charged into a $100 \mathrm{~mL}$ three-necked flask reactor containing $\mathrm{Fe}_{3} \mathrm{O}_{4}$ magnetic emulsion (total solid $1.4 \mathrm{gm}$ ). The reaction was carried out at $70^{\circ} \mathrm{C}$ with stirring rate 300 r.p.m. and under $\mathrm{N}_{2}$ atmosphere in the presence of the water-soluble initiator potassium persulfate (KPS). The reaction was allowed to proceed for $24 \mathrm{~h}$ and then terminated by cooling at room temperature. The prepared magnetic colloidal particles were filtered using glass wool fibers to remove any coarse particles before further analysis.

\subsubsection{Fabrication of Thin Films}

Colloidal particles were deposited on the substrate by layer by layer self-assembled multilayering using electrostatic interaction with oppositely charged polyelectrolytes. Polydimethyldiallylammonium chloride (PDAC), (Mw 100,000-200,000; 20\% aqueous solution) (Sigma-Aldrich) was used as cationic polyelectrolytes). Magnetic polymer colloidal dispersion of CSMPC with negative Zeta potential and size of $527 \mathrm{~nm}$ was employed as oppositely charges anionic solution. For a predetermined time, LBL-SAMU thin films were fabricated in 
custom made setup. Manually dipping the freshly cleaned glass slides (25.4mm X 76.2mm X 1mm) (Fischer) into the desired solutions of the opposite charge. Alternate layering was done to coat the prepared magnetic colloids (polymer core-shell magnetic nanoparticles total solids 1\%, size $527 \mathrm{~nm}$ ) in accordance to following steps; First adsorption stage: a charged substrate generally of negative charge is immersed for 10 minutes into the positively charged solution to be adsorbed on the surface of the slide for a known period, followed by first washing cycles to wash out unbound particles as shown in Figure 1. Each stage in the washing cycle takes about five minutes. Second adsorption stage: oppositely charged particles of the first adsorption stage were adsorbed on the substrate surface and then followed by the second washing cycle. The whole process constitutes about 40 min to get one bilayer. Repeating the protocol several times gives a multilayered structure for the desired thickness. The fabricated films were characterized by different techniques to study morphological properties and elemental composition, including scanning electron microscopy (SEM) and energy dispersive spectroscopy (EDS). (Jeol JSM 6490A) and optical microscopy (Optika B-600MET).

\subsection{Characterization of the epoxy-functionalized magnetic colloidal particles}

Before the application of the prepared magnetic colloidal particles (CSMPC) in MRI, they were characterized to determine the suitability of their use in biomedical diagnostic. The chemical composition of the prepared magnetic particles was examined by the Fourier-transform infrared (FTIR) spectroscopic technique using a Shimadzu FTIR 8400S instrument (IRPrestige-2, Japan). The spectra were recorded as the average of 40 scans in the region 400 to $4000 \mathrm{~cm}^{-1}$ at $4 \mathrm{~cm}^{-1}$ resolution. TEM (Phillips CM120) was used to investigate the morphology and microstructure of the magnetic polymer particles. Briefly, a drop of water diluted sample was deposited onto a carbon-coated copper grid and then left to dry at room temperature overnight before TEM imaging. Malvern Zetasizer 3000 HS instrument was used for measurement of average hydrodynamic size $\left(D_{\mathrm{h}}\right)$ of the magnetic polymer colloidal particles in $10^{-3} \mathrm{M} \mathrm{NaCl}$ solution, at $25^{\circ} \mathrm{C}$. The average value of three measurements was determined and taken into consideration. TGA (NETZSCH TG209) was used to determine the magnetite content of the magnetic polymer particles. The measurements were performed under a nitrogen atmosphere from ambient temperature up to $700^{\circ} \mathrm{C}$ with heating rate $20^{\circ} \mathrm{C} / \mathrm{min}$. The saturation magnetization (Ms) and magnetic behavior of the dried magnetic polymer latexes were investigated using a vibrating sample magnetometer (VSM). This test was carried out on the Automatic Bench of magnetic measurements, CNRS-IRC Lyon. The magnetization of all dried particles was investigated by decreasing the magnetic field $(\mathrm{H})$ from +20000 to -20000 Oersted, at room temperature. Malvern Zetasizer (NanoZS2000) was used to measure the charge density or zeta potential $(\zeta)$ of the magnetic polymer colloidal particles in $10^{-3} \mathrm{M} \mathrm{NaCl}$ solution at different $\mathrm{pH}$ media, at $25^{\circ} \mathrm{C}$. Each recorded value was the average of three measurements.

\section{In-Vitro Magnetic Resonance Imaging Studies}

Samples for MR-imaging studies were prepared by using variable concentrations (Fe concentration of 0.05 , $0.025,0.0125,0.00625,0.003125 \mathrm{ml} / 10 \mathrm{ml}$ of water) of CSMPC in deionized water. Testing was performed on all the samples using clinical $1.5 \mathrm{~T}$ Toshiba Vantage Titan scanner. $\mathrm{T}_{1}$ and $\mathrm{T}_{2}$ weighted images were captured during testing to measure both $\mathrm{T}_{1}$ sequences "Longitudinal relaxation time" (typically TR: 40, 225, 425, $825 \mathrm{~ms}$ and TE: $12 \mathrm{~ms}$ ) and $\mathrm{T}_{2}$ sequence "Transverse relaxation time" (typically TE: 15, 45, 75, 105, 135, $165 \mathrm{~ms}$ and TR: $5000 \mathrm{~ms}$ ). Experimentation was done under ambient conditions by placing the colloids directly on the top of the multichannel coil's body. MRI images were studied in K-Pacs-Lite and images were analyzed, visualized, and reconstructed using the statistical tool. Equal circular area (region of interest ROI) was chosen for each sample image and intensity data (Hounsfield) that is based on the intensity of the pixel contained within ROI were recorded. The mean intensity values were taken to compare relative intensity with reference (Deionized water) and each concentration.

\section{Results and discussion}

The synthesized magnetic colloids and fabricated thin films of colloids by LBL-SAMU were studied using different characterization techniques. The MRI studies along with characterization techniques are described below. 


\subsection{Characterization of the epoxy-functionalized magnetic colloidal particles}

Magnetic poly(divinylbenzene-co-glycidyl methacrylate) colloidal particles were successfully prepared using a seeded emulsion polymerization technique. The chemical composition of the prepared magnetic latex particles was investigated using FTIR spectroscopy (Figure 2). As clearly seen from the FTIR spectrum (Fig.2a), magnetic emulsion (ME) emulsion shows two characteristic peaks at 570 and $630 \mathrm{~cm}^{-1}$ which related to Fe-O vibration bands. After polymerization (Fig.2b), new absorption bands at $1600 \mathrm{~cm}^{-1}$ ( $\mathrm{C}=\mathrm{C}$ stretching) and $3000-3100 \mathrm{~cm}^{-1}(\mathrm{C}=\mathrm{CH}$ stretching $)$ of the aromatic benzene ring appeared, indicating the formation of poly(divinylbenzene) in presence of $\mathrm{Fe}_{3} \mathrm{O}_{4}$ nanoparticles. Additionally, a very characteristic peak at 1725 $\mathrm{cm}^{-1}\left(\mathrm{C}=\mathrm{O}\right.$ carbonyl vibration), two absorption bands at 1255 and $1180 \mathrm{~cm}^{-1}(\mathrm{C}-\mathrm{O}$ stretching) which related to the methacrylate ester vibrations of GMA units, and two characteristic peaks at 837 and $909 \mathrm{~cm}^{-1}(\mathrm{CH}$ stretching) of the oxirane ring of GMA also appeared. These results prove the successful copolymerization of the functional GMA monomer with DVB forming the epoxy-functionalized magnetic colloidal particles [2].

Morphology of the prepared magnetic polymer particles was investigated using TEM, as shown in Figure 3. TEM photos show the formation of well-defined core-shell morphology upon coating the magnetic emulsion droplets with the crosslinked poly(divinylbenzene-co-glycidyl methacrylate) copolymers. The average hydrodynamic diameter $\left(D_{\mathrm{h}}\right)$ of the prepared magnetic particles was measured using the DLS technique. After polymerization, the particle size of the magnetic emulsion droplets $(450 \mathrm{~nm})$ increased to be $527 \mathrm{~nm}$ due to the introduction of an organic part represented by the crosslinked poly(divinylbenzene-co-glycidyl methacrylate) polymer shell. Moreover, the prepared colloidal particles have uniform and narrow size distribution (i.e. monodisperse) as evidenced by polydispersity index $(\mathrm{PDI}=0.23)$ and TEM images

More interestingly, chemical structure, physical properties, and the amount of the used inorganic "magnetic" material in the final particle are the driven parameters controlling their physical separation under the magnetic field. Then, the good control of the magnetic properties before and after encapsulation is of paramount importance. In this regard, the magnetic content of the prepared magnetic latex particles was evaluated by using the TGA technique. As seen in Figure 4, TGA thermogram of $\mathrm{Fe}_{3} \mathrm{O}_{4}$ magnetic emulsion shows two main slopes; a very moderate one $\left(4 \%\right.$ weight loss) between 25 and $200{ }^{\circ} \mathrm{C}$, which attributed to release of moisture entrapped by the emulsified $\mathrm{Fe}_{3} \mathrm{O}_{4}$ nanoparticles, and a larger one (20\% weight loss) between 220 and $500{ }^{\circ} \mathrm{C}$ due to the decomposition of organic layer (oleic acid and SDS surfactants) treated $\mathrm{Fe}_{3} \mathrm{O}_{4}$ nanoparticles. This indicates that magnetic emulsion contains [?] $76 \%$ of magnetic iron nanoparticles. After polymerization of DVB and GMA, the magnetic content of the crosslinked poly(divinylbenzene-co-glycidyl methacrylate) coated $\mathrm{Fe}_{3} \mathrm{O}_{4}$ nanoparticles reduced to be [?] $66 \mathrm{wt} \%$ due to the incorporation of organic polymer shell during the polymerization process.

On the other hand, Figure 5 shows the magnetization curve of the magnetic emulsion (ME) before and after coating with poly(divinylbenzene-co-glycidyl methacrylate). As clearly seen from magnetization versus magnetic field (Fig. 5), the saturation magnetization of the seed magnetic emulsion (43.85 emu g-1) was reduced to be 35.49 emu. ${ }^{-1}$ after coating with poly(divinylbenzene-co-glycidyl methacrylate). More importantly, the superparamagnetic property was conserved which explains that the polymerization conditions have no significant effect on the intrinsic magnetic property of the used iron oxide nanoparticles.

Colloidal stability of the prepared magnetic polymer colloidal particles is of great interest especially when be

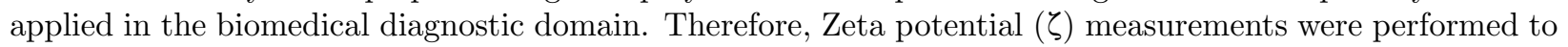
study the electro-kinetic phenomenon based on the surface charge of the prepared magnetic polymer particles in different $\mathrm{pH}$ environments. As shown in Figure 6, the prepared magnetic colloidal particles have a negative charge over the $\mathrm{pH}$ range investigated. In the case of magnetic emulsion seed (ME), the observed negative zeta potential, irrespective of the investigated $\mathrm{pH}$ domain, can be attributed to the negatively charged sulfate group $\left(\mathrm{SO}_{4}^{-}\right)$of SDS used during $(\mathrm{o} / \mathrm{w})$ magnetic emulsion formation. Regarding the prepared magnetic polymer particles, the negative zeta potential can be attributed to the negatively charged sulfate group $\left(\mathrm{SO}_{4}^{-}\right)$originating from the KPS initiator. Consequently, both systems (seed magnetic emulsion and prepared magnetic polymer particles) exhibit good colloidal stability over the $\mathrm{pH}$ range. 


\subsection{Characteristics of Fabricated Films}

\subsubsection{Fabrication of Self-assembled Thin Films}

In the current work, the LBL-SAMU technique presents several advantages over other techniques such as Langmuir Blodgett, organo-sulfur compounds chemisorbed on the metal surface, self-assembled monolayers, and multilayers of colloids. Relative to LBL-SAMU these techniques offer several problems in surface characterization control, the limitation for the material to be adsorbed, high yield surface reactions, and the molecules not firmly trapped. LBL-SAMU a typical technique involves the alternate layering of cationic and anionic charges on the substrate outer surface. It's a robust, stable, and environmentally friendly technique. Substrate topology and size does not affect the mechanism of the mentioned technique. Repeating the cycle several times, LBL-SAMU can be easily used to form multilayers with better control over the thickness, functionality, composition, uniformity, and molecular architecture [21-24].

\subsubsection{Morphology and growth studies of thin films}

Magnetic colloidal particles (with the average size of $527 \mathrm{~nm}$ ) were employed to prepare thin films bearing negative charge groups on their surface. Optika B-600MET optical microscopy (voltage range: 90V-240V $25 \mathrm{VA}-$ $50 / 60 \mathrm{~Hz}$ ) provides direct information about the uniformity of particle distribution, surface properties, and morphology. The representative optical microscopic micrographs of $5,10,15$ bilayers thin films are presented in Figure 7. An increasing trend for the particles adsorbed on the prepared substrate surface with an increased number of bilayers is obtained via self-assembly. Minimal particle's spreading was noticed on 5 bilayer micrograph due to less availability of charged ions. It is possible to happen that during alternate layering of cationic- anionic charges, adsorption may result in enhancement of deposition of particles [19, 21, $25,26]$. In addition, particle distribution appeared to be more and more uniform on the prepared substrate with increasing the number of bilayers.

To assess the constituting atoms relative composition within the synthesized biothin films of colloids, EDS analysis was done. The results of EDS analysis (Table 1) confirms that an increased number of bilayers provide large time for ions availability and their adsorption on the substrate that in turn leads to an increase in iron content. To obtain further insight into the growth of prepared biothin films and to support further optical micrographs, scanning electron microscopy (SEM) was performed. The SEM images of films reported that colloids particles may form agglomerates during adsorption. There are several regions where agglomerates are observed in SEM images. Figure 8 represents interesting morphology and growth patterns by SEM images. For example, a comparison of 5, 10 and 15 bilayers thin films depicting that colloid adsorption have significantly and gradually increased by increasing the number of bilayers with a $20 \%$ increment in iron contents imparting more magnetic characteristics. Particles are deposited as such in spherical geometry. Regarding the growth of films, most of the interesting information is significant in the present biothin film preparation technique. One can easily tune the parameters of this technique to control the thickness and magnetic characteristics imparted during the fabrication procedure. The fabricated films can find externally applied magnetic field tunablein vitro applications (e.g. therapy, and sensitive diagnostics such as protein DNA detection) [27].

\subsection{In-Vitro MRI of Magnetic Colloids}

MRI testing was performed to acquire two basic types of scan images based on $T_{1}$ and $T_{2}$ relaxation times. In MRI scanners, samples are placed under strong externally applied magnetic field area; an RF pulse is then applied to cause the protons of hydrogen atoms to spin with Larmour frequency and then relax after the removal of $\mathrm{RF}$ pulse. Both $\mathrm{T}_{1}$ and $\mathrm{T}_{2}$ images are different from each other in the sense that $\mathrm{T}_{2}$ is characterized by the transverse relaxation time (Spin-spin relaxation time), a time constant determining the signal decay, while $T_{1}$ is characterized by the longitudinal relaxation time (Spin-lattice relaxation time). The positive MR contrast agents (e.g. gadolinium) can reduce the spin-lattice relaxation time " $\mathrm{T}_{1}$ ". They are bright on MRI. It is observed that Gd is a well positive T1contrast agent, while colloids based on ferrofluids are a better 
negative $\mathrm{T}_{2}$ contrast agent. Both $\mathrm{T}_{1}$ and $\mathrm{T}_{2}$ relaxation mechanisms show different times for particular tissue in this way one can easily differentiate between tissues. Taking the importance of $\mathrm{T}_{1}$ and $\mathrm{T}_{2}$ images in MRI, magnetic colloids are studied for their potential to be used as MR contrast agents [10, 19, 28-32]. Table 2 summarized the results of the acquired $\mathrm{T}_{1}$ and $\mathrm{T}_{2}$ weighted images for their contrast performance. Mean intensity values are evident that the colloids in this study are relatively better performing as $M R \mathrm{~T}_{2}$ contrast agents. The $\mathrm{T}_{2}$ relaxation mechanism happens because of the energy exchange between the hydrogen atoms in water [30, 31, 33-35]. It seems that the signal intensities for each sample are not identical.

Contrast agents are the collection of contrast-enhancing media that are used in magnetic resonance imaging to increase contrast and to improve visualization of the internal body. After oral or intravenous administration of these agents, they interact with the atoms of the body and alter the relaxation times under the influence of a strong magnetic field. In MRI scanners during analysis; segments of the samples are exposed to a main (very strong) magnetic field. The relaxation process emits protons of energy that are detected by the scanner and recorded to get image after mathematical conversions.

The relaxation properties of the magnetic colloids were tested by measuring the $\mathrm{T}_{2}$ relaxation time of the water protons in the dispersion of colloids. The colloids create dephasing of protons moments and perturb the magnetic relaxation processes with shortened the water proton spin-spin relaxation times in the surrounding environment resulting in a reduced MRI signal intensity and consequent darkening of MR images. The resultant MR images ( $T_{1}$ and $T_{2}$ weighted) are presented in Figure 9.

Figure 10 exhibits the differences in contrast generation during $T_{2}$ and $T_{1}$ relaxation mechanisms. The MRI image can be acquired by different relaxation processes giving a lower, higher, or medium signal level. In the current work, it looks that $\mathrm{T}_{2}$ has strong relaxivity which points to a strong variation in contrast and signals intensity on $\mathrm{T}_{2}$ weighted images. Under the influence of externally applied higher magnetic field, to record the $\mathrm{T}_{2}$ relaxation time for each reported sample, ST: $5.1 \mathrm{~mm}$ were taken at various TE (echo time TE: $45,75,105,135$ and $165 \mathrm{~ms}$ ) with a repetition time (TR) of 5,000 ms. Similarly, the $\mathrm{T}_{1}$ relaxation time of reported samples was measured at TR: 40, 225, 425, and $825 \mathrm{~ms}$ while keeping constant echo time at $12 \mathrm{~ms}$. One more important observation is that with the increase in Fe concentration, MRI contrast becomes better that is due to the enhanced magnetic character within the sample. It might be a possible combination of iron oxides with functionalities that may cause an increase in hydrophilic character that allows nearer proximity of the protons with contrast agent molecules which leads to shorter spin-lattice relaxation time [36] [37]. Furthermore, with increased Fe concentration in colloidal suspension, the MR signal intensities are most significantly decreases giving steeper relaxation curve and providing dark manifestation by reduced spin-spin relaxation time. The variation in signal intensities with Fe concentrations also provide informational data about the relaxation rates due to energy exchange between the atoms. Since the MR contrast agents are those materials that are used to improve the visibility of body tissues during the scan. These agents can produce sufficient contrast to differentiate between normal area and diseased tissue by altering the relaxation time [34, 38-42]. This shows the efficient potential of the developed colloids as negative MR contrast agents.

\section{Conclusions}

Biopolymer core-shell magnetic colloids of the average size of $527 \mathrm{~nm}$ were successfully prepared. The prepared magnetic polymer particles have good colloidal stability and possess superparamagnetic properties which makes them suitable candidates to be used as contrast agents in magnetic resonance imaging (MRI). The detection and diagnostic applications of magnetic colloids were studied and both $\mathrm{T}_{1}$ and $\mathrm{T}_{2}$ weighted images were taken. The molecular imaging modalities showed that the colloids perform well as $\mathrm{T}_{2}$ contrast agents. With an increase in magnetic modalities within water dispersion, the contrast became darker, while the MR signal intensities decreased significantly forming a steeper relaxation curve. Fabrication of biothin films of the magnetic colloids was carried out effectively and colloids were adsorbed by retaining their characteristic properties to communicate with the biological systems. The film's growth was controlled by the number of bilayers deposited on the substrate. Particles formed agglomerates during adsorption. When comparing the bilayer thin films (5-15), $20 \%$ of iron contents became rise which may intensify the strength 
of magnetic character. Thus, our biopolymer core-shell magnetic colloids can be potentially developed with the magnetic functionality having significant medical applications, predominantly in diagnostic as well as in monitoring the effects of chemotherapy on tumor suppression.

\section{Acknowledgement}

This research was supported by the National University of Sciences and Technology, Islamabad, Pakistan (NUST).

\section{Conflict of interest}

There is no conflict of interest.

\section{References}

[1] Sun, C., Sze, R., Zhang, M., Folic acid-PEG conjugated superparamagnetic nanoparticles for targeted cellular uptake and detection by MRI. J. Biomed. Mater. Res. Part A 2006, 78, 550-557.

[2] Barick, K., Aslam, M., Prasad, P. V., Dravid, V. P., Bahadur, D., Nanoscale assembly of aminefunctionalized colloidal iron oxide.J. Magn. Magn. Mater. 2009, 321, 1529-1532.

[3] Hu, F., Jia, Q., Li, Y., Gao, M., Facile synthesis of ultrasmall PEGylated iron oxide nanoparticles for dual-contrast T1-and T2-weighted magnetic resonance imaging. Nanotechnol. 2011, 22 , 245604.

[4] Yamada, K., Sorensen, A. G., BASICS OF MRI.Neuro-oncology: The Essentials 2000, 56.

[5] Hornak, J. P., JP Hornak 1996.

[6] Martina, M.-S., Fortin, J.-P., Ménager, C., Clément, O., et al. , Generation of superparamagnetic liposomes revealed as highly efficient MRI contrast agents for in vivo imaging. J. Am. Chem. Soc. 2005, 127, 10676-10685.

[7] Eissa, M. M., Polymer Encapsulation of Magnetic Iron Oxide Nanoparticles for Biomedical Applications. J. Colloid Sci. Biotechnol. 2014, 3, 201-226.

[8] Naseer, N., Fatima, H., Asghar, A., Fatima, N., et al. , Magnetically Responsive Hybrid Polymer Colloids for Ultrasensitive Molecular Imaging. J. Colloid Sci. Biotechnol. 2014, 3 , 19-29.

[9] Hashemi, R. H., Bradley, W. G., Lisanti, C. J., MRI: the basics , Lippincott Williams \& Wilkins 2012.

[10] Rabias, I., Pratsinis, H., Drossopoulou, G., Fardis, M., et al. , In vitro studies on ultrasmall superparamagnetic iron oxide nanoparticles coated with gummic acid for T2 MRI contrast agent.Biomicrofluidics 2007, $1,044104$.

[11] Fanun, M., Colloids in drug delivery, CRC Press 2010.

[12] Rabias, I., Fardis, M., Devlin, E., Boukos, N., et al. , No aging phenomena in ferrofluids: the influence of coating on interparticle interactions of maghemite nanoparticles. ACS nano2008, 2 , 977-983.

[13] Zhong, Y., Whittington, C. F., Zhang, L., Haynie, D. T., Controlled loading and release of a model drug from polypeptide multilayer nanofilms. Nanomed- Nanotechnol., Bio. Med. 2007,3 , 154-160.

[14] Pankhurst, Q. A., Connolly, J., Jones, S., Dobson, J., Applications of magnetic nanoparticles in biomedicine. J. Phy. D: App. Phy. 2003, 36, R167.

[15] Ahmed, N., Ahmad, N. M., Fessi, H., Elaissari, A., In Vitro MRI of biodegradable hybrid (Iron oxide/polycaprolactone) magnetic nanoparticles prepared via modified double emulsion evaporation mechanism. Colloids Surface. B 2015. 
[16] Jada, A., A Special Issue on Inorganic Colloidal Particles, Synthesis, Surface Properties and Applications. J. Colloid Sci. Biotechnol. 2014, 3, 1-2.

[17] Gossuin, Y., Gillis, P., Hocq, A., Vuong, Q. L., Roch, A., Magnetic resonance relaxation properties of superparamagnetic particles. Wiley Interdisciplinary Reviews: Nanomed. Nanobiotechnol. 2009,1 , 299-310.

[18] Berry, C. C., Wells, S., Charles, S., Aitchison, G., Curtis, A. S., Cell response to dextran-derivatised iron oxide nanoparticles post internalisation. Biomater. 2004, 25 , 5405-5413.

[19] Hassan, M. A., Saqib, M., Shaikh, H., Ahmad, N. M., Elaissari, A., Magnetically Engineered Smart Thin Films: Toward Lab-on-Chip Ultra-Sensitive Molecular Imaging. J. Biom. Nanotechnol. 2013,9 , 467-474.

[20] Ahmad, N. M., Ali, S. J., Saqib, M., Stimuli-responsive self-assembled multilayer azo thin films: Effect of aggregates and salt on significant spectral shifts. J. Polym. Sci. Pol. Chem. 2012,50 , 1881-1889.

[21] Decher, G., Hong, J., Schmitt, J., Buildup of ultrathin multilayer films by a self-assembly process: III. Consecutively alternating adsorption of anionic and cationic polyelectrolytes on charged surfaces. Thin solid films 1992, 210 , 831-835.

[22] Arys, X., Fischer, P., Jonas, A. M., Koetse, M. M., et al. , Ordered polyelectrolyte multilayers. Rules governing layering in organic binary multilayers. J. Am. Chem. Soc. 2003, 125 , 1859-1865.

[23] Decher, G., Schlenoff, J. B., Multilayer thin films: sequential assembly of nanocomposite materials , John Wiley \& Sons 2006.

[24] Ulman, A., Formation and structure of self-assembled monolayers. Chem. Rev. 1996, 96 , 1533-1554.

[25] Ahmad, N. M., Saqib, M., Barrett, C. J., Novel Azobenzene-Functionalized Polyelectrolytes of Different Substituted Head Groups 1: Synthesis, Characterization and Absorption Spectroscopy Studies. J. Macromol. Sci. A 2009, 47, 106-118.

[26] Peyratout, C. S., Dahne, L., Tailor-made polyelectrolyte microcapsules: from multilayers to smart containers. Angewandte Chemie International Edition 2004, 43 , 3762-3783.

[27] Hafeli, U., Scientific and clinical applications of magnetic carriers, Springer 1997.

[28] Sinibaldi, E., Pensabene, V., Taccola, S., Palagi, S., et al. , Magnetic nanofilms for biomedical applications. J. Nanotechnol. Eng. Med. 2010, 1, 021008.

[29] Lee, D., Cohen, R. E., Rubner, M. F., Antibacterial properties of Ag nanoparticle loaded multilayers and formation of magnetically directed antibacterial microparticles. Langmuir 2005, 21 , 9651-9659.

[30] Laurent, S., Forge, D., Port, M., Roch, A., et al. , Magnetic iron oxide nanoparticles: synthesis, stabilization, vectorization, physicochemical characterizations, and biological applications. Chem. Rev. 2008, 108 , 2064-2110.

[31] Shultz, M. D., Calvin, S., Fatouros, P. P., Morrison, S. A., Carpenter, E. E., Enhanced ferrite nanoparticles as MRI contrast agents.J. Magn. Magn. Mater. 2007, 311, 464-468.

[32] Caravan, P., Strategies for increasing the sensitivity of gadolinium based MRI contrast agents. Chem. Soc. Rev. 2006,35, 512-523.

[33] Schweiger, C., Pietzonka, C., Heverhagen, J., Kissel, T., Novel magnetic iron oxide nanoparticles coated with poly (ethylene imine)- $<\mathrm{i}>\mathrm{g}</ \mathrm{i}>$-poly (ethylene glycol) for potential biomedical application: Synthesis, stability, cytotoxicity and MR imaging. Int. J. Pharm. 2011,408, 130-137.

[34] Jain, T. K., Richey, J., Strand, M., Leslie-Pelecky, D. L., et al. , Magnetic nanoparticles with dual functional properties: drug delivery and magnetic resonance imaging.Biomater. 2008, 29 , 4012-4021.

[35] Arsalani, N., Fattahi, H., Nazarpoor, M., Synthesis and characterization of PVP-functionalized superparamagnetic Fe3O4 nanoparticles as an MRI contrast agent. Exp. Polym. Lett. 2010,4, 329-338. 
[36] Jaganathan, H., Gieseck, R. L., Ivanisevic, A., Transverse relaxivity changes after layer-by-layer encapsulation of multicomponent DNA templated nanostructures. J. Phys. Chem. C 2010, 114 , 22508-22513.

[37] Rahman, M. M., Elaissari, A., Multi-stimuli responsive magnetic core-shell particles: synthesis, characterization and specific RNA recognition. J. Colloid Sci. Biotechnol. 2012, 1, 3-15.

[38] Mohammad-Taheri, M., Vasheghani-Farahani, E., Hosseinkhani, H., Shojaosadati, S. A., Soleimani, M., Fabrication and characterization of a new MRI contrast agent based on a magnetic dextran-spermine nanoparticle system. Iran. Polym. J. 2012, 21 , 239-251.

[39] Jaganathan, H., Gieseck, R. L., Ivanisevic, A., Characterizing proton relaxation times for metallic and magnetic layer-by-layer-coated, DNA-templated nanoparticle chains. Nanotechnol. 2010, 21 , 245103.

[40] Jaganathan, H., Hugar, D. L., Ivanisevic, A., Examining MRI contrast in three-dimensional cell culture phantoms with DNA-templated nanoparticle chains. ACS App. Mater. Interfaces 2011, 3 , 1282-1288.

[41] Zhang, Z., Sun, Q., Zhong, J., Yang, Q., et al. , Magnetic resonance imaging-visible and pH-sensitive polymeric micelles for tumor targeted drug delivery. J. Biomed. Nanotechnol. 2014,10 , 216-226.

[42] Wu, C., Lin, L., Lin, L., Huang, H., et al. , Biofunctionalized magnetic nanoparticles for in vitro labeling and in vivo locating specific biomolecules. App. Phys. Lett. 2008,92, 142504.

\section{Hosted file}

figures.docx available at https://authorea.com/users/328746/articles/455913-core-shellmagnetic-polymer-colloids-for-self-assembled-multilayers-thin-films-and-ultrasensitivemolecular-imaging

\section{Hosted file}

Tables.docx available at https://authorea.com/users/328746/articles/455913-core-shellmagnetic-polymer-colloids-for-self-assembled-multilayers-thin-films-and-ultrasensitivemolecular-imaging 